\title{
Microcephaly Outcomes among Zika Virus-Infected Pregnant Women in Honduras
}

Jackeline Alger, ${ }^{1,2,3} \dagger$ Pierre Buekens, ${ }^{4 \star} \dagger$ Maria Luisa Cafferata, ${ }^{5,6}$ Zulma Alvarez, ${ }^{7}$ Mabel Berrueta, ${ }^{5}$ Harry Bock, ${ }^{8}$ Carolina Bustillo, ${ }^{9,10}$ Alejandra Calderón, ${ }^{11}$ Allison Callejas, ${ }^{12}$ Mario Castillo, ${ }^{12}$ Alvaro Ciganda, ${ }^{6}$ Jenny Fúnes, ${ }^{12,13}$ Jorge García,,${ }^{1,2}$ Kimberly García, ${ }^{14}$ Luz Gibbons, ${ }^{5}$ Suzanne M. Gilboa, ${ }^{15}$ Emily W. Harville, ${ }^{4}$ Gustavo Hernández, ${ }^{16}$ Raquel López, ${ }^{2}$ Wendy López, ${ }^{1,2}$ Ivette Lorenzana, ${ }^{14}$ Marco Tulio Luque, ${ }^{17}$ Carlos Maldonado, ${ }^{18}$ Cynthia A. Moore, ${ }^{15}$ Carlos Ochoa, ${ }^{19}$ Leda Parham, ${ }^{14,20}$ Karla Pastrana, ${ }^{9,10}$ Fátima Rico, ${ }^{13,21}$ Heriberto Rodríguez, ${ }^{22}$ Candela Stella, ${ }^{5}$ Diana Valencia, ${ }^{15}$ Douglas Varela, ${ }^{23}$ Dawn M. Wesson, ${ }^{24}$ Concepción Zúniga, ${ }^{2,25}$ and Van T. Tong ${ }^{15}$

${ }^{1}$ Departamento de Laboratorio Clínico, Hospital Escuela, Tegucigalpa, Honduras; ${ }^{2}$ Instituto de Enfermedades Infecciosas y Parasitología Antonio Vidal, Tegucigalpa, Honduras; ${ }^{3}$ Unidad de Investigación Científica, Facultad de Ciencias Médicas, Universidad Nacional Autónoma de Honduras (UNAH), Tegucigalpa, Honduras; ${ }^{4}$ Department of Epidemiology, Tulane School of Public Health and Tropical Medicine, New Orleans, Louisiana; ${ }^{5}$ Instituto de Efectividad Clínica y Sanitaria, Buenos Aires, Argentina; ${ }^{6}$ Unidad de Investigación Clínica y Epidemiológica, Montevideo, Uruguay; ${ }^{7}$ Unidad de Vigilancia de la Salud, Región Sanitaria Metropolitana del Distrito Central (RSMDC), Secretaría de Salud de Honduras, Tegucigalpa, Honduras; ${ }^{8}$ Dirección General, RSMDC, Secretaría de Salud de Honduras, Tegucigalpa, Honduras; ${ }^{9}$ Departamento de Ginecología y Obstetricia, Hospital Escuela, Tegucigalpa, Honduras; ${ }^{10}$ Departamento de Ginecología y Obstetricia, Facultad de Ciencias Médicas, UNAH, Tegucigalpa, Honduras; ${ }^{11}$ Centro de Salud Alonso Suazo, RSMDC, Secretaría de Salud de Honduras, Tegucigalpa, Honduras; ${ }^{12}$ Servicio de Neonatología, Departamento de Pediatría, Hospital Escuela, Tegucigalpa, Honduras; ${ }^{13}$ Departamento de Pediatría, Facultad de Ciencias Médicas, UNAH, Tegucigalpa, Honduras; ${ }^{14}$ Centro de Investigaciones Genéticas, Instituto de Investigaciones en Microbiología, Facultad de Ciencias, UNAH, Tegucigalpa, Honduras; ${ }^{15}$ Division of Birth Defects and Infant Disorders, National Center on Birth Defects and Developmental Disabilities, Centers for Disease Control and Prevention, Atlanta, Georgia; ${ }^{16}$ Departamento de Pediatría, Hospital de Especialidades San Felipe, Tegucigalpa, Honduras; ${ }^{17}$ Servicio de Infectología, Departamento de Pediatría, Hospital Escuela, Tegucigalpa, Honduras; ${ }^{18}$ Servicio de Oftalmología, Departamento de Pediatría, Hospital Escuela, Tegucigalpa, Honduras; ${ }^{19}$ Servicio de Maternidad, Hospital de Especialidades San Felipe, Tegucigalpa, Honduras; ${ }^{20}$ Escuela de Microbiología, Facultad de Ciencias, UNAH, Tegucigalpa, Honduras; ${ }^{21}$ Departamento de Pediatría, Hospital Escuela, Tegucigalpa, Honduras; ${ }^{22}$ Sub-Dirección, Hospital de Especialidades San Felipe, Tegucigalpa, Honduras; ${ }^{23}$ Servicio de Neurología, Departamento de Pediatría, Hospital Escuela, Tegucigalpa, Honduras; ${ }^{24}$ Department of Tropical Medicine, Tulane School of Public Health and Tropical Medicine, New Orleans, Louisiana; ${ }^{25}$ Departamento de Vigilancia de la Salud, Hospital Escuela, Tegucigalpa, Honduras

Abstract. The impact of Zika virus (ZIKV) infection on pregnancies shows regional variation emphasizing the importance of studies in different geographical areas. We conducted a prospective study in Tegucigalpa, Honduras, recruiting 668 pregnant women between July 20, 2016, and December 31,2016. We performed Trioplex real-time reverse transcriptase-PCR (rRT-PCR) in 357 serum samples taken at the first prenatal visit. The presence of ZIKV was confirmed in seven pregnancies $(7 / 357,2.0 \%)$. Nine babies $(1.6 \%)$ had microcephaly (head circumference more than two SDs below the mean), including two $(0.3 \%)$ with severe microcephaly (head circumference [HC] more than three SDs below the mean). The mothers of both babies with severe microcephaly had evidence of ZIKV infection. A positive ZIKV Trioplex rRTPCR was associated with a $33.3 \%(95 \% \mathrm{Cl}: 4.3-77.7 \%)$ risk of $\mathrm{HC}$ more than three SDs below the mean.

The World Health Organization (WHO) Zika Causality Working Group's systematic review found sufficient evidence to conclude that Zika virus (ZIKV) is a cause of congenital abnormalities but with noted differences between countries. ${ }^{1}$ The increase in the number of cases of microcephaly potentially associated with ZIKV was large in Northeast Brazil in 2015. It was lower in other regions of Brazil and in other countries. ${ }^{2,3}$ The reason for these variations is unknown and emphasizes the importance of studying the impact of ZIKV infection in different geographical areas, including Central America. Phylogenetic evidence suggests that Zika virus (ZIKV) arrived in Honduras in $2015,{ }^{4}$ but the epidemic occurred in 2016, when a total of 32,132 suspected cases of ZIKV infections were recorded, including 10,386 cases from the Tegucigalpa metropolitan area (Región Metropolitana del Distrito Central). ${ }^{5}$ At the national level, the number of reported cases was the highest at the beginning of 2016, reflecting an initial concentration of ZIKV infections in the San Pedro Sula area (Cortes Department, Atlantic coast). ${ }^{4,6}$ In the Tegucigalpa metropolitan area, the number of reported cases was at its height in mid-2016 (Figure 1), ${ }^{5}$ reflecting the season with the

*Address correspondence to Pierre Buekens, W. H. Watkins Professor of Epidemiology School of Public Health and Tropical Medicine, Tulane University, 1440 Canal St., Suite 2001, New Orleans, LA 70112. E-mail: pbuekens@tulane.edu

†These authors contributed equally to this work. highest risk of mosquito-borne disease transmission, as illustrated by previous studies on circulation of dengue virus (DENV) and chikungunya virus (CHIKV). ${ }^{7}$

We conducted a prospective pregnancy cohort study in Tegucigalpa, Honduras: the Zika in Pregnancy in Honduras (ZIPH) study to investigate the impact of congenital ZIKV infection. The ZIPH protocol was previously published. ${ }^{8} \mathrm{We}$ enrolled pregnant women at their first prenatal visit and followed up them through delivery. At the time of enrollment, women were interviewed to collect sociodemographic data. Data about ZIKV symptoms during pregnancy were collected at the first visit and at delivery. Infant head circumference $(\mathrm{HC})$ at birth and gestational age estimated by last menstrual period, prenatal ultrasound, clinical examination, or Capurro scores $^{9}$ were used to classify microcephaly. Using the INTERGROWTH-21st standards for gender and gestational age, microcephaly at birth was defined as $\mathrm{HC}$ more than two $\mathrm{SD}$ selow the mean; severe microcephaly was defined as $\mathrm{HC}$ more than three SDs below the mean. ${ }^{10,11}$ Clinical data, such as neuroimaging, were abstracted from available medical records for those infants born to women with positive ZIKV by real-time reverse transcriptase-PCR ( $r R T-P C R)$ results. Enrollment into the cohort started in July 2016 and continues. The current analysis is among pregnant women enrolled until December 31, 2016, during the end of the ZIKV outbreak in Honduras.

Venous maternal blood was collected once as soon as possible after enrollment at the first prenatal visit, regardless 


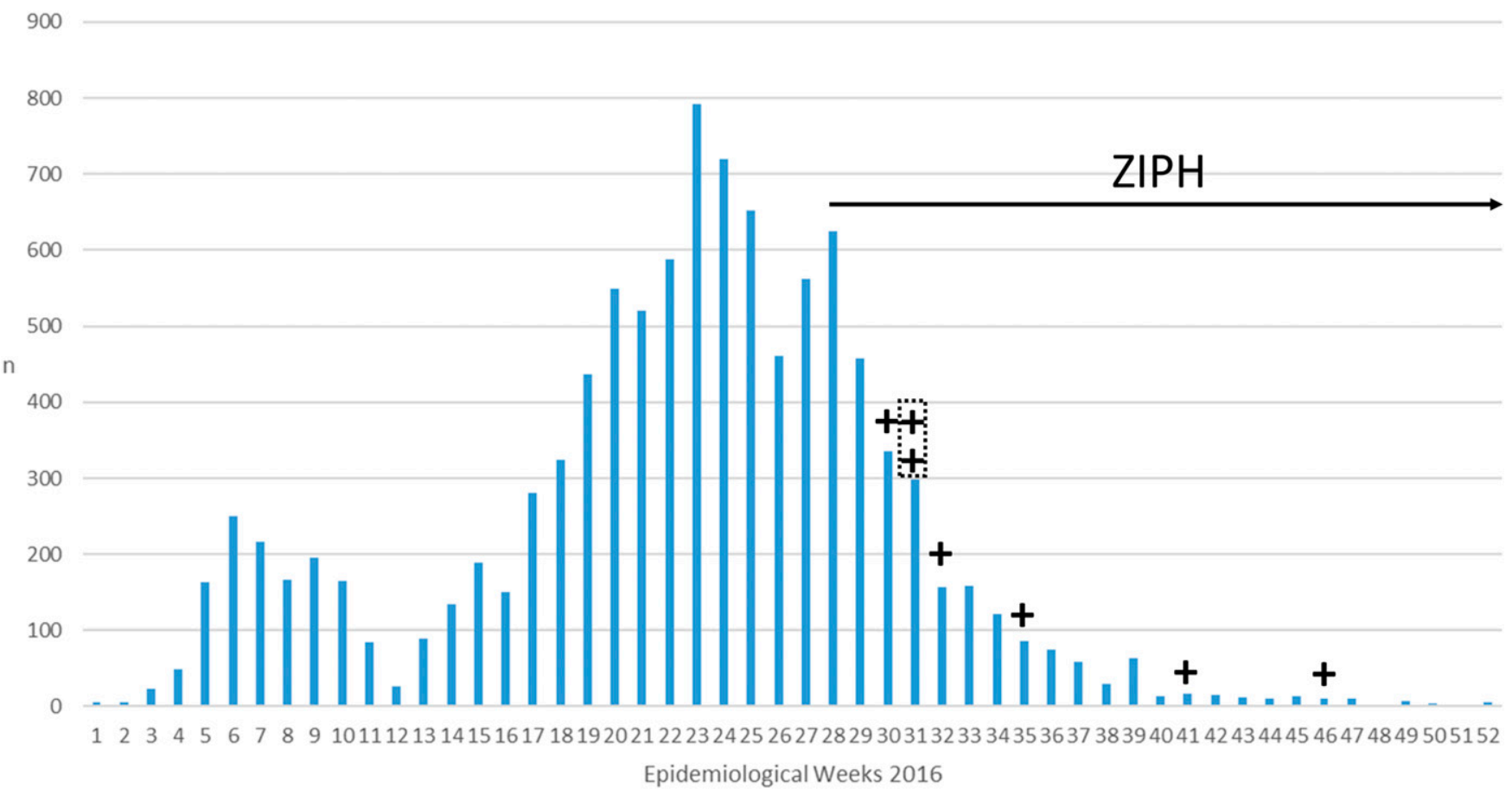

FIGURE 1. Number $(n)$ of suspected cases of ZIKV infections reported in the Tegucigalpa metropolitan area (Región Sanitaria Metropolitana del Distrito Central), January-December 2016 (epidemiological weeks 1-52). ${ }^{5}$ The Zika in Pregnancy in Honduras (ZIPH) study started on week 28. Crosses (+) denote Trioplex rRT-PCR assays positive for ZIKV among ZIPH participants. Crosses in a dotted line box denote both rRT-PCR assay and head circumference more than three SDs below the mean. rRT-PCR = real-time reverse transcriptase-PCR; ZIKV = Zika virus. This figure appears in color at www.ajtmh.org.

of symptoms. Blood samples were initially frozen at or below $-20^{\circ} \mathrm{C}$ and later at or below $-80^{\circ} \mathrm{C}$ until testing. We conducted molecular testing for ZIKV, DENV, and CHIKV on maternal serum samples using the Trioplex rRT-PCR assay, which detects RNA from all three viruses simultaneously, following CDC protocols. ${ }^{12,13}$ Consent forms include an opt-out provision that otherwise allows participating study institutions to store blood specimens for up to 10 years. No laboratory testing was performed on infants.

We computed the frequency of ZIKV infection at the first prenatal visit among enrolled women ("exposed women"). We also computed the frequency of $\mathrm{HC}$ more than two SDs and more than three SDs below the mean in infants. We calculated the risk for microcephaly in exposed and unexposed infants with the corresponding Fisher exact 95\% Cls. We used $R$ version 4.0.0 for data analysis. This study was approved by the Tulane University and the Faculty of Medical Sciences, Universidad Nacional Autónoma de Honduras institutional review boards.

All 705 women attending prenatal care at Alonso Suazo Health Center between July 20, 2016 and December 31, 2016 were screened for enrollment; 668 provided written informed consent and were recruited from the center (Supplemental Material, Figure S1). The first prenatal visit occurred before 14 weeks of gestation in $62.8 \%$ of women (Table 1). Seventeen women reported symptoms compatible with ZIKV infection during pregnancy. We collected information from medical records of 644 (96.4\%) women at the end of their pregnancy, including 599 live births, 36 pregnancy losses, and nine stillbirths. The gestational age at birth was based on the last menstrual period $(82.6 \%, 516 / 625)$, ultrasound $(14.2 \%$, $89 / 625)$, clinical examination $(3.7 \%, 23 / 625)$, or Capurro score $(0.2 \%, 1 / 625)$. The proportion of preterm deliveries ( $<37$ weeks) was $15.5 \% ; 11.5 \%$ of pregnancies resulted in a low-birthweight newborn $(<2,500 \mathrm{~g})$ (Table 1). Nine babies $(1.6 \%)$ had microcephaly, including two $(0.3 \%)$ with severe microcephaly (Table 2). Figure 1 shows that the mothers of babies with severe microcephaly were recruited in early August 2016, at the very beginning of the recruitment period.

Data collected from medical records suggested that the two babies with severe microcephaly had Zika-associated birth defects (see Supplemental Material). The first newborn had a malformed skull with a narrow forehead and a cerebral tomography showing ventriculomegaly, agenesis of the corpus callosum, and multiple calcifications. The second newborn had a malformed skull with a prominent occiput, and a cerebral tomography with ventriculomegaly and multiple calcifications.

A blood sample was taken at the first prenatal visit in 667 recruited women (Supplemental Figure S1). Almost half of the participants $(45.8 \%, 306 / 667)$ did not consent to their blood samples being stored long term to permit testing when resources would potentially become available. We performed rRT-PCR among 357 women who consented to the long-term storage of their blood samples (Supplemental Figure S1). Table 1 shows that the characteristics of the women with and without consent to store samples were similar. Of those who consented, seven $(7 / 357,2.0 \%)$ were ZIKV positive (Table 2$)$. Two of the women with ZIKV-positive results had symptoms during pregnancy (Supplemental Material, Table S1). Figure 1 shows that four of the ZIKV-positive mothers were recruited at the very beginning of the study. Both mothers of babies with severe microcephaly were ZIKV positive. No other newborn born to mothers with positive ZIKV had microcephaly (Table 2). A positive ZIKV test at the first prenatal visit $(n=6)$ was 
TABLE 1

Maternal and neonatal characteristics among women with and without serum samples tested by Trioplex rRT-PCR assay ZIPH study, Honduras, 2016

\begin{tabular}{|c|c|c|c|c|c|c|}
\hline & \multicolumn{2}{|c|}{ All women $(N=667)$} & \multicolumn{2}{|c|}{ rRT-PCR performed $(N=357)$} & \multicolumn{2}{|c|}{ No rRT-PCR performed $(N=310)$} \\
\hline & $n / N^{*}$ & $\%$ & $n / N$ & $\%$ & $n / N$ & $\%$ \\
\hline \multicolumn{7}{|l|}{ Maternal age-group (years) } \\
\hline $12-19$ & 198/667 & 29.7 & $91 / 357$ & 25.5 & $107 / 310$ & 34.5 \\
\hline 20-34 & $404 / 667$ & 60.6 & $233 / 357$ & 65.3 & $171 / 310$ & 55.2 \\
\hline 35 or older & $65 / 667$ & 9.7 & $33 / 357$ & 9.2 & $32 / 310$ & 10.3 \\
\hline Years of education and range $\dagger$ & 9 & $6-12$ & 9 & $6-12$ & 9 & $6-11$ \\
\hline \multicolumn{7}{|l|}{ Gestational age at the first visit (weeks) } \\
\hline Between one and 13 & $374 / 596$ & 62.8 & $198 / 322$ & 61.5 & $176 / 274$ & 64.2 \\
\hline Between 14 and 27 & $176 / 596$ & 29.5 & $97 / 322$ & 30.1 & $79 / 274$ & 28.8 \\
\hline 28 or older & $46 / 596$ & 7.7 & $27 / 322$ & 8.4 & $19 / 274$ & 6.9 \\
\hline \multicolumn{7}{|l|}{ Symptoms during pregnancy } \\
\hline Skin rash & $12 / 644$ & 1.9 & $7 / 353$ & 2.0 & $5 / 291$ & 1.7 \\
\hline Fever & $15 / 644$ & 2.3 & $8 / 353$ & 2.3 & $7 / 291$ & 2.4 \\
\hline Arthralgia & $11 / 644$ & 1.7 & $5 / 353$ & 1.4 & $6 / 291$ & 2.1 \\
\hline Arthritis & $3 / 644$ & 0.5 & $2 / 353$ & 0.6 & $1 / 291$ & 0.3 \\
\hline Conjunctivitis & $5 / 644$ & 0.8 & $2 / 353$ & 0.6 & $3 / 291$ & 1.0 \\
\hline None & $626 / 644$ & 97.2 & $343 / 353$ & 97.2 & $283 / 291$ & 97.3 \\
\hline Preterm birth (less than 37 weeks) & $97 / 625$ & 15.5 & $54 / 348$ & 15.5 & $43 / 277$ & 15.5 \\
\hline Low birthweight $(<2,500 \mathrm{~g})$ & $68 / 592$ & 11.5 & $30 / 329$ & 9.1 & $38 / 263$ & 14.4 \\
\hline Very low birthweight $(<1,500 \mathrm{~g})$ & $11 / 592$ & 1.9 & $3 / 329$ & 0.9 & $8 / 263$ & 3.0 \\
\hline Microcephaly $(\mathrm{HC}<2 \mathrm{SD})$ & $9 / 578$ & 1.6 & $6 / 323$ & 1.9 & $3 / 255$ & 1.2 \\
\hline Severe microcephaly $(\mathrm{HC}<3 \mathrm{SD})$ & $2 / 578$ & 0.3 & $2 / 323$ & 0.6 & $0 / 255$ & 0.0 \\
\hline \multicolumn{7}{|l|}{ Newborn status } \\
\hline Alive & $599 / 644$ & 93.0 & $332 / 353$ & 94.1 & $267 / 291$ & 91.8 \\
\hline Still birth & $9 / 644$ & 1.4 & $4 / 353$ & 1.1 & $5 / 291$ & 1.7 \\
\hline Pregnancy loss & $36 / 644$ & 5.6 & $17 / 353$ & 4.8 & $19 / 291$ & 6.5 \\
\hline
\end{tabular}

$\mathrm{HC}=$ head circumference; $r R T-P C R=$ real-time reverse transcriptase-PCR

${ }^{*}$ The denominators presented reflect the number of participants with information in the ZIPH study.

$\dagger$ Median [interquartile range].

associated with a $33.3 \%(95 \% \mathrm{Cl}: 4.3-77.7)$ risk of severe microcephaly, whereas the risk was 0 (95\% Cl: 0-1.2) in 317 unexposed.

Our results show an association between confirmed ZIKV infection in the first half of pregnancy and severe microcephaly. Both cases of severe microcephaly were recruited at the beginning of the study, close to the estimated peak of the epidemic in Honduras (Figure 1), and were born in January and February 2017. This is consistent with monthly reports from the largest hospital in Tegucigalpa showing an increase of cases of microcephaly from July 2016 to February 2017, with a peak of 11 cases per month in December 2016 and January 2017, compared with one case or less per month before July 2016. ${ }^{14}$ The two cases of severe microcephaly in the current study were not sufficient to substantially increase the overall rates of microcephaly. We observed similar lower than expected rates of microcephaly in Uruguay during 2010-2015. ${ }^{11}$

\section{TABLE 2}

Zika virus (ZIKV) rRT-PCR results and microcephaly, ZIPH study, Honduras, 2016

\begin{tabular}{|c|c|c|c|c|c|c|c|}
\hline \multirow[b]{2}{*}{ Head circumference } & \multicolumn{2}{|c|}{ ZIKV + } & \multicolumn{2}{|c|}{ ZIKV- } & \multicolumn{2}{|c|}{$\begin{array}{l}\text { No rRT-PCR } \\
\text { performed }\end{array}$} & \multirow[b]{2}{*}{ Total } \\
\hline & $n / N$ & $\%$ & $n / N$ & $\%$ & $n / N$ & $\%$ & \\
\hline Microce & $2 / 6$ & 33.3 & $4 / 317$ & 1.3 & $3 / 255$ & 1.2 & \\
\hline Severe & $2 / 2$ & 100 & $0 / 4$ & - & $0 / 3$ & - & - \\
\hline No microcephaly & $4 / 6$ & 66.6 & $313 / 317$ & 98.7 & $252 / 255$ & 98.8 & 569 \\
\hline Not measured & 1 & & 33 & - & 55 & - & 8 \\
\hline Total & 7 & & 350 & - & 310 & - & 667 \\
\hline
\end{tabular}

rRT-PCR = real-time reverse transcriptase-PCR; ZIKV = Zika virus.
The official data on reported ZIKV cases (Figure 1) are based on suspected cases, often not confirmed by laboratory analyses. Both over- and underreporting are possible, but the shape of the time distribution is most likely accurate. We previously published plaque reduction neutralization test results confirming that the ZIPH study population was exposed to ZIKV. ${ }^{15}$

In the United States, the risk of ZIKV-associated birth defects was $15 \%$, with laboratory-confirmed ZIKV infection during the first trimester; however, the case definition was broader and included brain abnormalities identified with neuroimaging and eye abnormalities based on ophthalmological examination. ${ }^{16,17}$ The risk of microcephaly among infected women is estimated to be $4-6 \%$ globally. ${ }^{18} \mathrm{~A}$ recent systematic review found high heterogeneity among studies, with microcephaly risk among infected women varying from $0.9 \%$ to $30 \% .^{19}$ In our study, $33.3 \%(95 \% \mathrm{Cl}: 4.3-77.7)$ of women with a positive ZIKV test at the first prenatal visit delivered an infant with microcephaly. Both cases of severe microcephaly were born to mothers whose blood was drawn during the first half of their pregnancies (13 and 16 weeks of gestation), in agreement with the literature showing that the risk of Zika-associated birth defects is higher when the mother is infected by ZIKV at the beginning of pregnancy. ${ }^{20}$ Only one of the two mothers was symptomatic, which is consistent with the literature suggesting that risk is similar for ZIKV infection regardless of the presence of symptoms. ${ }^{19}$

Our study was initially designed as a case-cohort study to limit laboratory analyses, ${ }^{8}$ but additional funds allowed us to test women recruited in 2016. Samples from women who did not agree for their blood sample to be stored for more than 1 year after delivery could not be tested, but characteristics of 
women with and without rRT-PCR were similar. Another limitation of the ZIPH study is that we only collected a blood sample at the first prenatal visit. Despite the limitations of the study and the small numbers, the simplified protocol of the ZIPH study allowed us to initiate the study quickly, at the peak of the ZIKV epidemic in Tegucigalpa. We conclude that ZIKV infection in early pregnancy is associated with severe microcephaly in Honduras as observed in other regions of the Americas.

Received November 17, 2020. Accepted for publication January 19, 2021.

Published online March 15, 2021.

Note: Supplemental information, table, and figure appears at www. ajtmh.org.

Acknowledgments: We thank Karla Rivera and Renato Valenzuela for their administrative support. We thank Andrea Meyer for editing this manuscript.

CDC Disclaimer: The findings and conclusions in this report are those of the authors and do not necessarily represent the official position of the CDC.

Financial support: The Zika in Pregnancy in Honduras (ZIPH) study is partially funded by Vysnova Partners SC-2018-3045-TU.

Authors' addresses: Jackeline Alger, Jorge García, and Wendy López, Departamento de Laboratorio Clínico, Hospital Escuela Universitario, Tegucigalpa, Honduras, and Instituto de Enfermedades Infecciosas y Parasitología Antonio Vidal (IAV), Tegucigalpa, Honduras, E-mails: jackelinealger@gmail.com, jalgar62_84@yahoo.com.ar and wlopez36@ hotmail.com. Pierre Buekens, Emily W. Harville, and Dawn M. Wesson, Tulane University School of Public Health and Tropical Medicine, New Orleans, LA, E-mails: pbuekens@tulane.edu, eharvill@tulane.edu, and wesson@tulane.edu. Maria Luisa Cafferata, Mabel Berrueta, Luz Gibbons, and Candela Stella, Instituto de Efectividad Clínica y Sanitaria (IECS), Buenos Aires, Argentina, E-mails: marialuisa.cafferata@gmail. com, mberrueta@iecs.org.ar, lgibbons@iecs.org.ar, and cstella@iecs. org.ar. Zulma Alvarez and Harry Bock, Región Sanitaria Metropolitana del Distrito Central, Secretaría de Salud de Honduras, Tegucigalpa, Honduras, E-mails: zulmahen@yahoo.com and hbockme@hotmail.com. Carolina Bustillo and Karla Pastrana, Departamento de Ginecología y Obstetricia, Facultad de Ciencias Médicas, UNAH, Tegucigalpa, Honduras, E-mails: mcbu1502@yahoo.com and kpastrana_2000@yahoo. com.mx. Alejandra Calderón, Centro de Salud Alonso Suazo, Región Sanitaria Metropolitana del Distrito Central, Secretaría de Salud de Honduras, Tegucigalpa, Honduras, E-mail: lilianalecalderon@gmail.com. Allison Callejas, Mario Castillo, and Jenny Fúnes, Servicio de Neonatología, Departamento de Pediatría, Hospital Escuela, Tegucigalpa, Honduras, E-mails: amariecs1981@gmail.com, mariocastillo26@yahoo. com, and jennylagosfunes@yahoo.com. Alvaro Ciganda, Unidad de Investigación Clínica y Epidemiológica, Montevideo, Uruguay, E-mail: aciganda@gmail.com. Kimberly García, Ivette Lorenzana, and Leda Parham, Facultad de Ciencias, UNAH, Tegucigalpa, Honduras, E-mails: kimfa_2010@hotmail.com, ivettelorenzana@yahoo.com, and Iparham29@ hotmail.com. Suzanne M. Gilboa, Cynthia A. Moore, Diana Valencia, and Van T. Tong, Division of Birth Defects and Infant Disorders, National Center on Birth Defects and Developmental Disabilities, Centers for Disease Control and Prevention, Atlanta, GA, E-mails: suz0@cdc.gov, cam0@cdc.gov, ile9@cdc.gov, and vct2@cdc.gov. Gustavo Hernández, Carlos Ochoa, Heriberto Rodríguez, Hospital de Especialidades San Felipe, Tegucigalpa, Honduras, E-mails: ghernandezbustillo@yahoo.es, caof@email.com, and mmfhrg@gmail.com. Raquel López, Instituto de Enfermedades Infecciosas y Parasitología Antonio Vidal, Tegucigalpa, Honduras, E-mail: raquelbonilla_86@hotmail.com. Marco Tulio Luque, Carlos Maldonado, Fátima Rico, Douglas Varela, Departamento de Pediatría, Hospital Escuela, Tegucigalpa, Honduras, E-mails: mtluque@ yahoo.com, cmcolegiaciones@gmail.com, ricourrea7@gmail.com, and douglasvarela2068@gmail.com. Concepción Zúniga, Departamento de Vigilancia de la Salud, Hospital Escuela, Tegucigalpa, Honduras, E-mail: concepcionzuniga@gmail.com.

\section{REFERENCES}

1. Krauer F, Riesen M, Reveiz L, Oladapo OT, Martínez-Vega R, Porgo TV, Haefliger A, Broutet NJ, Low N, Group WZCW, 2017. Zika virus infection as a cause of congenital brain abnormalities and guillainBarré syndrome: systematic review. PLoS Med 14: e1002203.

2. de Oliveira WK, de França GVA, Carmo EH, Duncan BB, de Souza Kuchenbecker R, Schmidt Ml, 2017. Infection-related microcephaly after the 2015 and 2016 Zika virus outbreaks in Brazil: a surveillance-based analysis. Lancet 390: 861-870.

3. Ospina ML et al., 2020. Zika virus disease and pregnancy outcomes in Colombia. N Engl J Med 383: 537-545.

4. Metsky HC et al., 2017. Zika virus evolution and spread in the Americas. Nature 546: 411-415.

5. Secretaría de Salud, Honduras, 2016. Boletin Epidemiologico. Tegucigalpa, Honduras: Republica de Honduras. Available at: http://www.salud.gob.hn/site/index.php/vigilancia.

6. Zhang Q et al., 2017. Spread of Zika virus in the Americas. Proc Natl Acad Sci U S A 114: E4334-E4343.

7. Zambrano LI, Sierra M, Lara B, Rodríguez-Núñez I, Medina MT, Lozada-Riascos CO, Rodríguez-Morales AJ, 2017. Estimating and mapping the incidence of dengue and chikungunya in Honduras during 2015 using Geographic Information Systems (GIS). J Infect Public Health 10: 446-456.

8. Buekens $P$ et al., 2016. Zika virus infection in pregnant women in Honduras: study protocol. Reprod Health 13: 82.

9. Capurro H, Konichezky S, Fonseca D, Caldeyro-Barcia R, 1978. A simplified method for diagnosis of gestational age in the newborn infant. J Pediatr 93: 120-122.

10. Villar J et al.; (INTERGROWTH-21st) IFaNGCftsC, 2014. International standards for newborn weight, length, and head circumference by gestational age and sex: the newborn cross-sectional study of the INTERGROWTH-21st project. Lancet 384: 857-868.

11. Harville EW, Buekens PM, Cafferata ML, Gilboa S, Tomasso G, Tong V, 2020. Measurement error, microcephaly prevalence and implications for Zika: an analysis of Uruguay perinatal data. Arch Dis Child 105: 428-432.

12. Centers for Disease Control and Prevention, 2017. Trioplex Real-Time rT-PCR Assay: Instructions for Use. Available at: https://www.cdc.gov/zika/pdfs/trioplex-real-time-rt-pcr-assayinstructions-for-use.pdf. Accessed May 15, 2020.

13. Santiago GA et al., 2018. Performance of the Trioplex real-time RT-PCR assay for detection of Zika, dengue, and chikungunya viruses. Nat Commun 9: 1391.

14. Hospital Escuela, 2017. Semana epidemiologica 22, 28 de Mayo 03 de Junio 2017. Tegucigalpa, Honduras: Hospital Escuela.

15. Ward MJ et al., 2018. Zika virus and the World Health Organization criteria for determining recent infection using plaque reduction neutralization testing. Am J Trop Med Hyg 99: 780-782.

16. Honein MA, 2018. Recognizing the global impact of Zika virus infection during pregnancy. N Engl J Med 378: 1055-1056.

17. Reynolds MR et al., 2017. Vital signs: update on Zika virusassociated birth defects and evaluation of all U.S. Infants with congenital Zika virus exposure - U.S. Zika pregnancy registry, 2016. MMWR Morb Mortal Wkly Rep 66: 366-373.

18. Musso D, Ko Al, Baud D, 2019. Zika virus infection - after the pandemic. N Engl J Med 381: 1444-1457.

19. Ximenes R, Ramsay LC, Miranda RN, Morris SK, Murphy K, Sander B, Team RLR, 2019. Health outcomes associated with Zika virus infection in humans: a systematic review of systematic reviews. BMJ Open 9: e032275.

20. Gallo LG et al., 2020. Another piece of the Zika puzzle: assessing the associated factors to microcephaly in a systematic review and meta-analysis. BMC Public Health 20: 827. 